\title{
Personalised Nudging for more Data Disclosure? On the Adaption of Data Usage Policies Format to Cognitive Styles
}

\author{
Charlotte Schöning \\ LMU Munich \\ schoening@bwl.lmu.de
}

\author{
Christian Matt \\ University of Bern \\ christian.matt@iwi.unibe.ch
}

\author{
Thomas Hess \\ LMU Munich \\ thess@bwl.lmu.de
}

\begin{abstract}
While highly sensitive data like personal health information (PHI) is valuable to digital health service providers, users often remain reluctant to disclose such personal data. Research has shown that personalised nudging, i.e. nudging that adopts content to user characteristics to nudge specific actions, can successfully increase purchase intention. However, its effect on consumers' handling of sensitive data is unclear. We apply personalised nudging in the context of personalising data usage policies and investigate whether personalised nudges that match users' cognitive styles (i.e. the way users process information), affects individuals' level of trust, privacy concerns, risk, and PHI disclosure. Using an online experiment in the context of mobile apps for health bonus programmes, we find that, when presentation format matches the users' cognitive style individuals' PHI disclosure and trust are not affected, but that individuals' privacy concerns and risk perceptions are significantly lower.
\end{abstract}

\section{Introduction}

As consumers and users of digital products and services, we are constantly faced to make decisions in an online environment. In business and economics studies research has long relied on the assumption that an individual will always make a pure cost-benefit calculation when facing a decision. However, this also implies that an individual has perfect information which we know from reality is impossible. Humans have cognitive limitations and their decisions underlie different biases. For example, the same issue can be expressed through text or pictures resulting in different perceptions of the content among individuals. That means it is also important how a choice is presented and that this presentation format can also "nudge" the user towards a decision. Nudging, or in an online environment, digital nudging, means guiding people's online choices by distinct interface design elements [63].

One of the decisions we constantly make in an online environment is for example how much personal data we disclose to digital service providers. Privacy concerns generally inhibit users from disclosing their personal data [1, 2]. In addition, as per law, websites and apps must provide data usage policies that state what consumer data is collected for which purpose, but these policies often fail to alleviate consumers' privacy concerns, or might even trigger them [3, 4]. This decision is even more difficult when it comes to personal health information as it is highly sensitive. One example for such a case are so-called health bonus programmes, offered by many European health insurance providers to their insured to encourage and incentivise both a healthier lifestyle and regular attendance of medical check-ups in exchange of monetary benefits, e.g. a free treatment or a cash bonus. Users can monitor their behaviour and progress through the programme-corresponding app which requires the users to disclose their PHI. User interfaces of those apps can be designed in a way to nudge people to disclose their PHI. It is important to note however, that nudging is to be separated from manipulation, as the goal of nudging is to benefit both the user and the provider which is the case for these health insurance apps. Keeping up a healthier lifestyle will benefit the users, as they are less prone to get ill, will maintain fitness, and thus can prevent diseases. Yet, it will also benefit the health insurance company, as it can save costs when their clients are healthier and therefore less in need of insurance claims.

Not only practitioners but also IS scholars have become increasingly interested in the (theoretical) examination of digital nudging [e.g. 44, 47, 53, 63], especially exploring the importance of user interface design. However, the underlying principle of digital nudging is generally a one-size-fits all approach, which is also the case for the communication of privacy or security messages $[38,53]$. Yet, research has shown that people process information differently, which means that a digital nudge should be most effective 
when it is tailored to the individual user. The tailoring is based for example on personal preferences the user has previously expressed through behaving in a distinct manner online. This so-called personalised nudging is becoming increasingly relevant in practice. Tailoring has been studied in human computer interaction literature and persuasion literature [49], but it has yet rarely been studied in the digital nudging literature [44]. Recent work in privacy research showed that presentation format is an important factor of personalisation [27] and that distinct wordings correlate with different personality traits [38]. Moreover, we know from marketing research that purchase intention can be increased by matching the website design to the users' preferred way to process information, i.e. their cognitive style [22]. However, personalised nudging in the context of data usage policies is different, because here, sensitive data need to be disclosed. Gaining a first understanding of the effect of personalised nudges in a privacy context is especially relevant, as personalisation as such can raise users' privacy concerns about data disclosure [10, 33, 58]. Therefore, we aim to close this research gap by investigating the effect of personalised nudges on data disclosure. As users are especially solicitous in regard to highly sensitive personal data like personal health information, these provide the most interesting setting for studying personalised nudges in the privacy context [9]. Moreover we want to investigate the effect of personalised nudges on individuals' privacy perceptions, i.e. on trust, privacy concerns, and risk. These three constructs have been shown to be especially important when it comes to privacy-related decisions [18, 55]. Therefore, we ask the following research questions:

RQ1: How do data usage policies that match users' cognitive style influence their perception on trust, privacy concerns, and risk?

RQ2: How do data usage policies that match users' cognitive style influence their level of PHI disclosure?

We chose the case of an app for health bonus programmes provided by health insurance companies as an appropriate application case to investigate the combination of cognitive styles and presentation format as a form of a personalised nudge in the context of data usage policies regarding personal health information. Our work expands the scarce body of knowledge on personalised nudging and provides practitioners with valuable insights about how this specific nudging technique can steer users towards a more favourable evaluation of their products or services.

The paper proceeds as follows: We will give an overview of the essential theoretical concepts and the related literature. We will then present our research model and develop the hypotheses. After that, we will outline our research method before analysing and discussing the results. We will infer both theoretical contributions and managerial implications from the findings and conclude with a brief synopsis.

\section{Theoretical foundations}

\subsection{Digital nudging}

In general, a nudge is defined as "any aspect of the choice architecture that alters people's behavior in a predictable way without forbidding any options or significantly changing their economic incentives" [60, p. 6]. The underlying idea of nudging is based on insights from behavioural economics and psychology research which demonstrated that when it comes to making decisions, individuals do not act rationally but rather irrationally, as they underlie biases and heuristics [e.g. 26, 54]. This stands in contrast to the previously prevalent depiction of an individual in classic economics as homo economicus, having unbounded rationality, unbounded willpower and unbounded pursuit of self-interest. It is important to note that the original idea of nudging is not a purely paternalistic and manipulative approach, but that the freedom of choice is remained [60]. In the public sector, the concept of nudging has been used in the past years to steer individuals towards decisions that benefitted themselves and consequently, society, for example nudging people towards a more environmentally-friendly behaviour.

As more and more decisions are nowadays made in an online environment, IS scholars have transferred the concept of nudging into the digital sphere [2, 44, 47, 63]. Consequently, digital nudging is defined as "the use of user-interface design elements to guide people's behavior in digital choice environments" [63, p. 433]. Hence, the concept of digital nudging is about suppressing or exploiting biases and heuristics in an online choice environment, to steer people into towards distinct decisions. Like "normal" nudging, digital nudging can be used to achieve a plethora of goals, ranging from paying $\mathrm{CO}_{2}$ offsets while booking flights [59] to increasing online social sharing [25], and, as mentioned above, to nudging privacy [4]. While having its roots in the public sector, the private sector has also commenced to exploit nudging, and digital nudging in particular, for their purposes. Firstly, digital nudging is both relatively cheap and easy to implement [63]. Secondly, due to increasingly elaborate tracking techniques and steadily improving computing power, nudging online offers new possibilities related to personalisation. 


\subsection{Personalised nudging and cognitive styles}

Personalised nudging is a form of digital nudging that takes into account users' individual characteristics and behaviour patterns. It is important to note that the presentation of choices is personalised, not the choices themselves, i.e. freedom of choices is ensured. Personalisation of nudges can be done based on the users' cognitive style. An individual's cognitive style is defined as "a person's preferred way of gathering, processing, and evaluating information" [23, p. 850]. Studies across disciplines like psychology, marketing, and consumer behaviour have exhibited that individuals differ in cognitive style and that this is relevant in the decision-making process $[15,38]$. There are different dimensions of cognitive styles $[6,20,22$, 39 , 52]. A widely agreed upon distinction in psychology, marketing, and education is the dimension "visual-verbal" $[19,30,41,51]$. Therefore, the visualverbal dimension will also be our focal dimension. While individual search or purchasing behaviour is widely used for the personalisation of web content, cognitive styles are yet rarely used.

Consequently, website and app interfaces can be designed in a way that they match a user's cognitive style to achieve specific goals. Hauser et al. [22] showed that in a sales context, matching a website's presentation format to the user's cognitive style lead to an increase in purchase intention. Here, the website designs differed for instance in the amount of data presented and if information was displayed using additional graphs. In the privacy context, personalised nudging can usually serve two contradictory purposes, namely assisting privacy and the contrary, which has been described in the privacy literature as "nudging away from privacy" $[3,5,11,61]$. In the privacy context, Malkin et al. [38] studied the efficacy of different wordings of browser warnings, which were developed to appeal to different personality traits, on user behaviour. Keith et al. [27] investigated the effect of different presentation formats on trust and risk perceptions as well as disclosure behaviour in regard to privacy. However, there has not yet been conducted a study that investigates the effect of the congruence of presentation format and personal characteristics.

\subsection{Personal health information: privacy perceptions and disclosure behaviour}

The GDPR which recently came into force in the European Union defines personal health information as "personal data related to the physical or mental health of a natural person, including the provision of health care services, which reveal information about his or her health status" [1, p. 33, Art. 4]. PHI can for example include chronic diseases, mental health conditions, or information about reproductive status $[28,32]$. In the course of the ongoing digitalisation covering all areas of life, more and more technologies using PHI have entered the market. On the one side there are digital developments like the much discussed electronic health record, maintained and professionally used by health care providers [13]. On the other side, a steady growing market for products like consumer health wearables and fitness apps has emerged, where personal health information is collected and analysed by companies unrelated to the health care sector [e.g. 13, 34]. A significant problem here is that the advertising industry but also health insurance companies have already shown third-party interest in getting access to this kind of data.

A widely used model in privacy research to depict individuals' privacy-related perceptions and behaviour is the APCO model (Antecedents, Privacy Concerns, Outcomes) by Smith et al. [55] and its enhanced version by Dinev et al. [18]. This model is also applicable in the context of personal health information. Three constructs are central in the model, influencing user behaviour. These constructs are trust, privacy concerns, and risk. Risk is usually displayed together with benefits to form the so-called privacy calculus. However, we concentrate on risk only as we are interested in why people refrain from an action rather than in which benefits they see. Moreover, risk perception, not the perceived benefit, is influenced by the privacy concerns [18]. Risk is assessed by users as the "perceived risk of opportunistic behavior related to the disclosure of personal information submitted" [24, p. 298]. For the second construct we take into consideration, trust, manifold definitions exist. Related to our research, the definition of Mishra [48] is highly appropriate. Following Mishra, trust can be defined as "willingness to be vulnerable to another party based on the belief that the latter party is 1) competent, 2) open, 3) concerned, and 4) reliable" [48, p. 5]. Lastly, we review users' privacy concerns. For defining privacy concerns, we draw on the work by Hong \& Thong to construe them as "the degree to which an Internet user is concerned about website practices related to the collection and use of his or her personal information" [24, p. 276]. Users display privacy concerns when it comes do disclosing personal data, and those are especially high when it comes to disclosing personal health information, as it is highly sensitive $[9,14,18$, $55]$. 


\section{Conceptual model and hypotheses development}

Building on the aforementioned theoretical insights we developed a conceptual model depicted in Figure 1. The model considers three stages. First, we determine if presentation format matches the user's cognitive style or not. This is our independent variable. Second, we predict that a match has a positive effect on users' privacy perception (the second stage in the model) as well as on their behaviour, i.e. the level of PHI disclosure.

As stated above, data usage policies have been demonstrated to often be too long and complex [42, 61]. Tsai et al. [61] proved that when privacy information of online vendors is made available in a concise and compact way, users will choose vendors that better protect their privacy, underpinning the importance of presentation format for digital nudging. Moreover, research has shown that people differ in the way they process information, i.e. they differ in their cognitive style. In IS research, the role of personal characteristics has been studied in the context of online retail [29, 43, 62]. More specifically, the literature stream in IS on privacy has examined the importance between personal characteristics and perceived privacy $[12,27,38]$. We assume that matching presentation format and cognitive style will influence users' perception of privacy. Two concepts from psychology are especially relevant here, namely familiarity and processing fluency [e.g. 21, 31, 36, 57, 64]. Processing fluency refers to the phenomenon "that people tend to prefer easily processed information" [7, p. 9369].
When individuals try to understand complicated information, they rely on such mechanisms [7]. Based on these insights, we assume that if users are shown information in a way that matches their cognitive style, they process this information more easily.

Combining these insights we assume that matching the presentation format to the cognitive style of the user will first enhance understanding because of a better processing fluency. Furthermore, fluent stimuli are assessed as more familiar by individuals [7]. Existing literature indicates that familiarity will then in turn have an effect on trust [21,31]. Thus, we suggest that when users are shown the data usage policy in their preferred way to process this information, the understanding of the content will be better than when it is displayed otherwise. In line with the literature, we expect that the better understanding will lead to a higher level of trust. Accordingly, we predict:

H1. Users will show higher levels of trust when presentation format matches their cognitive style.

The central variable of the APCO model are privacy concerns. An individual's privacy concerns are determined by antecedents such as privacy awareness. Privacy awareness describes the degree to which a user understands how the collected personal information is used [24, 37]. We assume that a matching of the presentation with the users' cognitive style will enhance the users' understanding of the content and awareness of how it is used. Research has also shown that being aware of an issue and understanding it thoroughly lowers concerns [50]. Consequently, we presume:

H2. Users will show lower levels of concerns when presentation format matches their cognitive style.

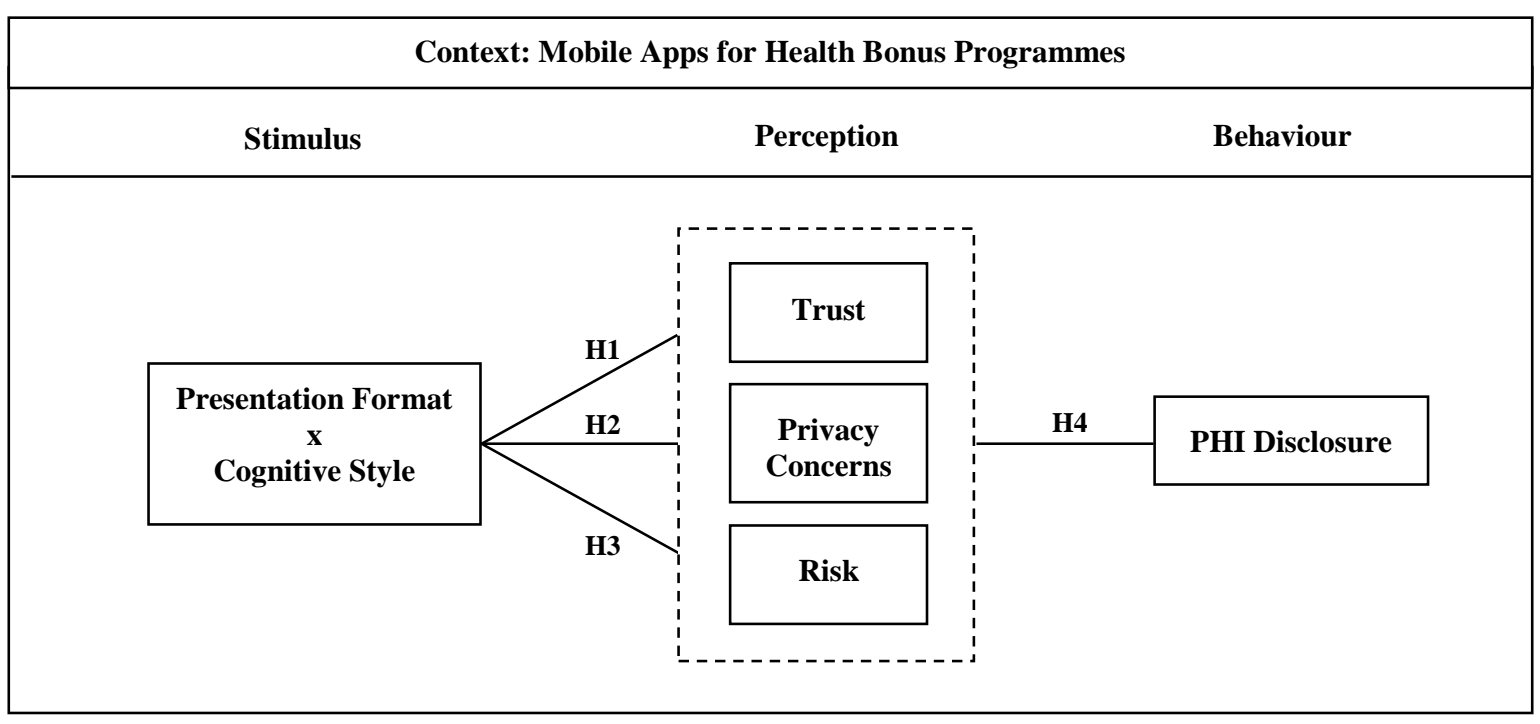

Figure 1. Conceptual model 
The third central construct when it comes to information privacy and data disclosure are risk beliefs. Although there are subcategories of risk [45], we concentrate on risk in general for simplification reasons. The level of perceived risk is determined by how a user assesses the likelihood of opportunistic behaviour by the digital service provider regarding the disclosed personal information [24]. The assessment of risk is an intuitive process and heavily influenced by processing fluency [57]. A stimulus is perceived as unfamiliar when processing fluency is low which leads to an increased risk perception [57]. We derive from this insight that correspondingly, when we have a high processing fluency, familiarity will increase, which then in turn has an alleviating effect on user's risk perception. Thus, for reducing the risk perceived by the user, processing fluency has to be enhanced which we assume can be achieved by matching the presentation format of a website with the users' cognitive style. Hence, we expect consumers, who are shown the data usage policy in a design that matches their cognitive style, to express a lower level of perceived risk than consumers who are shown a non-matching version. We thus hypothesise as follows:

H3. Users will show lower levels of risk when presentation format matches their cognitive style.

Besides users' perceptions of trust, concerns, and risk, also their behaviour can be influenced by the congruence of presentation format and cognitive style. As Hauser et al. [22] show, presenting products of a website in a way that matches the user's preferred way to process information, can enhance purchase intention. Transferring these findings to our context, we assume that PHI disclosure as behavioural reaction can be increased. This corresponds to the findings by Alter \& Oppenheimer [8], which show that fluency promotes self-disclosure. Consistent with the aforementioned argumentation we expect participants in the match group to have a higher level of PHI disclosure than participants in the no group. Hence, we predict:

H4. Users will show higher levels of PHI disclosure when presentation format matches their cognitive style.

\section{Research method}

\subsection{Experimental design}

To test our hypotheses, we developed an experimental set-up in the context of a health bonus programme by a health insurance company and its respective app as described above. Bonus points are awarded e.g. for having successfully achieved a set fitness goal or for undergoing the yearly dental check. In addition, those apps often offer additional services, for example a tool to track one's medication etc. If individuals have collected a specified amount of points within one year, they are given a cash bonus or free treatments. As users' disclosure of PHI provides benefits for both the user and the provider, this setting provides a highly suitable case for our research goal. When developing the case and the designs, we followed the concept of a real-world bonus programme by a large German health insurance company to create an experience as real as possible.

We conducted an online experiment, using a scenario-based questionnaire created with Qualtrics. Building on the insights from previous research about digital nudging, interface design, privacy policies, and cognitive styles, we developed two different types of nudges. These two nudges served as our treatments representing the two poles of the aforementioned "visual-verbal" dimension. Based on the findings by Tsai et al. [61] that compact summary versions work better than lengthy data usage policies, we decided to develop two summaries with a different presentation formats of data usage policies each. This also suits the app case, as apps have limited screen space. Presentation format 1 showed the summary displaying the information about data usage with icons. Presentation format 2 showed the summary displaying the information with bullet points. Each presentation format caters for the visual respective the verbal type. Although we will evaluate the results only based on the two different groups of "match" and "no match", we chose to work with two presentation formats (visual and verbal), to ensure that an effect is not only due to a certain presentation format, e.g. visual, as would be the case if we had only worked with one presentation format, e.g. icons. Participants were randomly assigned to one of the two nudges. Respondents were recruited via a university database and via Facebook; in total, the questionnaire was 100 percent completed by 156 individuals. The study was conducted among Germanspeaking participants, thus all explanations and questions were translated into German. In our set-up participants were told to receive $100 €$ for 1000 points achieved in one year.

After having received the treatment, respondents were to answer a questionnaire covering our constructs of interest. Firstly, participants had to answer question regarding the three constructs for privacy perception, trust, concerns, and risk. Secondly, for determining the respective cognitive style, i.e. if someone classified a visual or a verbal type, we employed three semantic differentials with questions drawn from the literature [e.g. 30, 56]. Finally, respondents had to fulfil a disclosure task where they were asked to provide 
personal health information (with the additional possibility of "not specified"). It is important to note, that the nudges (i.e. the data usage policies) are personalised, but regarding the decision, the experiment is in line with the original idea of nudging of retaining the freedom of choice in the choice architecture, namely the decision how much PHI is disclosed.

The treatments and the questions underwent thorough examinations in a pretest. We controlled for medication, i.e. if people are currently taking medication which is only available on prescription by providing three possible answers, namely "yes", "no", and "not specified". Finally, we controlled for different demographics.

\subsection{Operationalisation of constructs}

To operationalise our constructs, we extracted validated measures from relevant existing literature and adapted them to our case. The construct of trust and its items have been derived from Dinev \& Hart [17] and Malhotra et al. [37]. The items were adjusted and modified to suit our case. The items for concerns were drawn from Kenny \& Connolly [28], who had adapted the items by Hong \& Thong [24] to the health context and combined them with items by $\mathrm{Li}$ et al. [35]. For measuring the perceived risk we used the items by Dinev \& Hart [17] for "perceived Internet privacy risk", and adjusted them to the PHI context. Items for trust and concerns were measured on a 7point Likert scale, ranging from "strongly disagree" to "strongly agree". Perceived risk was to be ranked from "no risk" to "very high risk", also using a 7-point Likert scale. As stated in our hypotheses we presume that these three constructs influence the level of PHI disclosure. For determining the level of PHI disclosure we asked "I have or had...", followed by different types of conditions. These were, e.g. chronic disease, cancer, mental health condition, miscarriage or abortion, and surgery, taken from [32] and [28]. Participants were able to select multiple answers. Moreover we included a "none of the above"-option and a "not specified"-option. We then created a dummy variable for PHI disclosure aggregating the answers of the participants regarding the conditions. Regarding the three semantic differentials for determining the users' cognitive style, respondents had to rate the following statements on a 6-point Likert scale. First, "for comprehension, I find figures rather helpful/not helpful", second, "I am rather the visual/verbal type", and third, "when I need to understand facts, I prefer explanations in form of images/text". After aggregating the three scales, participants with a cumulated average under 3.5 (i.e. the middle of the scale) classified as visual (127 participants), participants with a cumulated average greater than 3.5 classified as verbal (29 participants).

\section{Results}

First, we conducted a factor analysis using SPSS, for assessing factor loadings and reliability measures. For trust and concerns, all items scored satisfactorily, showing communalities above 0.5 and factor loadings above 0.7 [40]. For risk, two items did not meet the required criteria of showing communalities above 0.5 and factor loadings above 0.7 to ensure validity. Thus, they were excluded from further analysis [40]. However, three items of the construct "risk" met the required criteria, leaving us with a satisfactory valid factor. Factor loadings for items of all factors were between 0.837 and 0.937. Next, we calculated Cronbach's Alpha values for the remaining items. All items showed a sufficiently high Cronbach's Alpha value greater than 0.7 , thus ensuring reliability [16]. For all three constructs, mean values were between 4.39 and 5.2 and standard deviation between 1.31 and 1.69 .

For determining the difference between the "yes" group (match, 75 participants) and the "no" group (no match, 81 participants), we conducted a t-test for independent samples with SPSS. First, the difference in the level of trust between the groups is not significant $(\mathrm{p}=0.080)$. Thus, H1 is not supported. Second, the results show that, as predicted, the level of concerns is significantly lower in the yes group than in the no group, to wit on the 0.05 level $(\mathrm{p}=0.017)$. Therefore, $\mathrm{H} 2$ is supported. Our third variable regarding privacy perception, risk, shows a significant effect for the difference in perceived risk between the yes group and the no group. Significant on the 0.01 level $(p=0.006)$,

Table 1. Results of the t-test

\begin{tabular}{|c|c|c|c|c|}
\hline Construct & $\begin{array}{c}\text { Match } \\
\mathbf{y}(1) \\
\mathbf{n}(\mathbf{0})\end{array}$ & Mean & $\begin{array}{l}\text { Std. } \\
\text { Err. }\end{array}$ & $\begin{array}{l}(1-0) \\
\text { Mean } \\
\text { difference }\end{array}$ \\
\hline \multirow{2}{*}{ Trust } & 1 & 4.75 & 0.16 & \multirow{2}{*}{$0.39^{\text {ns }}$} \\
\hline & 0 & 4.36 & 0.15 & \\
\hline \multirow{2}{*}{ Concerns } & 1 & 4.56 & 0.17 & \multirow{2}{*}{$-0.53^{*}$} \\
\hline & 0 & 5.09 & 0.14 & \\
\hline \multirow{2}{*}{ Risk } & 1 & 4.68 & 0.14 & \multirow{2}{*}{$-0.55^{* *}$} \\
\hline & 0 & 5.23 & 0.14 & \\
\hline \multirow{2}{*}{$\begin{array}{l}\text { PHI } \\
\text { disclosure }\end{array}$} & 1 & 0.52 & 0.06 & \multirow{2}{*}{$-0.02^{\mathrm{ns}}$} \\
\hline & 0 & 0.54 & 0.06 & \\
\hline \multicolumn{5}{|c|}{$* * \mathbf{p}<0.01$} \\
\hline
\end{tabular}


participants in the yes group show significantly lower risk perception than participants in the no group. Hence, we can also support H3. The results do not show significant difference in the level of PHI disclosure between the two groups, thus we were not able to support H4. Lastly, the control variables did not show any effects.

\section{Discussion}

\subsection{Key findings}

The results of the study offer rich insights into the effects of congruence between cognitive styles and presentation format on users' privacy perceptions and their disclosure behaviour for PHI. Consistent with our expectations, we were able to show that matching users' cognitive style with an interface's presentation format significantly decreases users' levels of risk and concerns. This means, users are less concerned about the provider's practices regarding collecting and using the data when presentation format and cognitive style correspond. Moreover, users perceive the risk that the digital service provider, in our case the health insurance company, will engage in opportunistic behaviour regarding the disclosed personal (health) information, to be lower when we have a congruence of cognitive style and presentation format. That is because the congruence will increase the understanding of the content, as processing fluency is better. Fluent stimuli are then in turn evaluated as more familiar [7, 64]. Hence, the identified effects are based on the two concepts of familiarity [21, 31, 64] and fluency [7, 8, 57].

Surprisingly, we were not able to support $\mathrm{H} 1$ regarding the level of trust. As the influence of a match was most significant for perceived risk, and generally a decrease in risk leads to an increase in trust and vice versa, this is unexpected [12, 17]. A possible explanation could be that referring to our definitions of the constructs, trust is a much stronger perception than risk or privacy concerns, due to the willingness of being vulnerable. Therefore, users might not react to the nudge as quickly, because they actually reflect about the decision. Therefore, fluency might be undermined to a certain extent. However, we acknowledge that the $\mathrm{p}$ value of 0.080 was only marginally not classified as significant at the 0.05 level. This indicates that significance might increase with a larger sample size.

The other result that contradicted our expectation was that matching cognitive style with presentation format did not have any effect on the level of PHI disclosure. In contrast to $\mathrm{H} 1$ however, the $\mathrm{p}$ value of
0.773 was not near any significance level. Although previous literature has shown that processing fluency has an influence of both perception and behaviour, the outcomes of this experiment show that perceptions are influenced, but not behaviour. One possible explanation for this outcome is, that it is relatively easy to express perceptions, but actually expressing behaviour always comes with a certain risk. Thus, the latter might require more effort to change. Moreover, in our derivation of $\mathrm{H} 4$, we have drawn on insights that show that when products of a website are presented in a way that matches the user's preferred way to process information, purchase intention is enhanced [22]. The fact that we could not find such an effect in our context of sensitive data suggests, that this "riskiness" in behaviour might be even greater regarding actions related to sensitive information. This also indicates there are hitherto unknown forces or cognitive mechanisms between the perception level and the behavioural level that are yet to be discovered.

\subsection{Theoretical contributions and managerial implications}

The findings of the study provide important implications for both theory and practice. Our work contributes to IS research in several ways. First, it expands the steadily growing literature on digital nudging and the scarce literature on personalised nudging in particular. We were able to show that matching presentation format with the user's cognitive style can alleviate users' concerns and risk. This stresses the importance for IS research for further investigations on the role of cognitive styles for personalised nudging (see also [38]). For example, the results provide a sound basis for investigating different dimensions of cognitive styles concerning digital nudging and specifically personalised nudging. Our findings also contribute to the privacy literature and especially the relationship between privacy perceptions and behavioural outcomes, as depicted by the APCO model. Here, our results hint at the fact that the influential factors on perception and behaviour, as well as the relationships between the constructs of trust, concerns, and risk, differ in their intensity. Moreover the constructs, especially behaviour might be affected by additional cognitive mechanisms.

The results of the experiment also provide various managerial implications, for both digital health service providers and digital service providers in general. While we were not able to show that disclosure behaviour can be increased, our findings nevertheless indicate that digital health service providers should consider to use personalised nudging. Disclosure 
behaviour might not be directly affected, however, as we were able to show that concerns and risks can be significantly decreased when employing personalised nudging. Thus, personalised nudging equips practitioners with a tool to design their interfaces in a way that alleviates concerns and risks towards their product or company. Moreover, although personalised nudging might not directly influence users' disclosure behaviour, it can steer users towards a more favourable evaluation of the provider's product or service. In the long term, this might have also positive effects on the adoption of the product or service and might consequently indirectly lead to an increased disclosure behaviour.

\subsection{Limitations and future research}

There are certain limitations of the study which simultaneously open various avenues for further research. First, we chose an app's interface for the reasons stated above. However, the possibilities for designing a mobile interface are limited because of fewer space available. Thus, it would be interesting to conduct similar studies with a desktop interface and to compare the results. Second, although the concept of different cognitive styles is widely agreed upon, the distribution of the types might not be equally distributed among the population. For example, our sample turned out to be comprised of circa three quarters of visual type and one quarter of verbal type. Regarding determining the different types, the selfreporting bias might also be problematic. Therefore, we urge scholars to further investigate the relationship between cognitive styles and presentation format. For example, other dimensions of cognitive styles can be examined. Third, the setting of the experiment might have been another limitation. The experiment was conducted in the name of a university. This might have biased participants' PHI disclosure behaviour as universities are generally perceived to be respectable and to handle personal data in a secure fashion, so participants did not have to fear consequences of their disclosure. This might be different with a real health insurance company, which indicates that $\mathrm{H} 4$ might be supported in the real world. Hence, a study in collaboration with a company might be helpful for further insights in this regard. Fourth, our sample consisted of German students. Therefore, it would be interesting to conduct a generational and/or crosscultural study, as there might be differences between age groups or between cultures, indicated for example by the study of Miltgen and Peyrat-Guillard [46]. Fifth, there might be other effects influencing perception and/or behaviour, which are beyond the scope of this paper. In a follow-up study, e.g. the type of disease and possible associated feelings could be included as a moderator. Finally, it would be interesting to investigate to which point or extent people are "easy" to nudge or if there was something like a "resistance to nudges".

Our results indicate that besides trust, concerns, and risk, there are additional mechanisms at play between the perception level and the behaviour level. Referring to the enhanced APCO model by Dinev et al. [18], these mechanisms might be triggered by the level of effort that moderates the relationships between the constructs of perception and the behavioural reactions. However, there might be also mechanisms which have yet to be discovered. To conclude, after the provision of initial insights in this study, much more research has to be done for a thorough understanding of the role of cognitive styles regarding personalised nudging.

\section{Conclusion}

This study aimed to expand the literature on user interface design and personalised digital nudging by examining the effect of the congruence of presentation format and users' cognitive style on users' PHI disclosure behaviour. To investigate this effect, we conducted an online experiment. The findings illustrate that cognitive style does not assist in nudging people towards a higher level of PHI disclosure or trust. However, the fact that the outcomes showed a significant effect on risk and concerns demonstrates that cognitive styles play a significant role in users' privacy perceptions and thus, influences users' judgment in Internet-based systems. We believe that this area of research unfolds a plethora of interesting research avenues and encourage scholars especially from the field of IS, but also from related disciplines, to engage in studying personalised nudging.

\section{References}

[1] "EU General Data Protection Regulation (GDPR): Regulation (EU) 2016/679 of the European Parliament and of the Council of 27 April 2016 on the protection of natural persons with regard to the processing of personal data and on the free movement of such data, and repealing Directive 95/46/EC (General Data Protection Regulation), OJ 2016 L 119/1."

[2] Acquisti, A., "Nudging privacy: The behavioral economics of personal information", IEEE Security \& Privacy, 7(6), 2009,

[3] Acquisti, A., Adjerid, I., Balebako, R., Brandimarte, L., Cranor, L.F., Komanduri, S., Leon, P.G., Sadeh, N., Schaub, F., and Sleeper, M., "Nudges for privacy and security: Understanding and assisting users' choices online", ACM Computing Surveys (CSUR), 50(3), 2017, pp. 44. 
[4] Acquisti, A., Adjerid, I., Balebako, R., Brandimarte, L., Cranor, L.F., Komanduri, S., Leon, P.G., Sadeh, N., Schaub, F., Sleeper, M., Wang, Y., and Wilson, S., "Nudges for Privacy and Security: Understanding and Assisting Users' Choices Online", ACM Computing Surveys, 50(3), 2016,

[5] Acquisti, A., and Grossklags, J., "Privacy and Rationality in Individual Decision Making", IEEE Security \& Privacy, 3(1), 2005, pp. 24-30.

[6] Allinson, C.W., and Hayes, J., "The Cognitive Style Index: A Measure of Intuition-Analysis for Organizational Research", Journal of Management Studies, 33(1), 1996, pp. 119-135.

[7] Alter, A.L., and Oppenheimer, D.M., "Predicting shortterm stock fluctuations by using processing fluency", Proceedings of the National Academy of Sciences, 103(24), 2006, pp. 9369-9372.

[8] Alter, A.L., and Oppenheimer, D.M., "Uniting the tribes of fluency to form a metacognitive nation", Personality and social psychology review, 13(3), 2009, pp. 219-235.

[9] Angst, C.M., and Agarwal, R., "Adoption of Electronic Health Records in the Presence of Privacy Concerns: The Elaboration Likelihood Model and Individual Persuasion", MIS Quarterly, 33(2), 2009, pp. 339-370.

[10] Awad, N.F., and Krishnan, M.S., "The Personalization Privacy Paradox: An Emprical Evaluation of Information Transparency and the Willingness to be Profiled Online for Personalization", MIS Quarterly, 30(1), 2006, pp. 13-28.

[11] Balebako, R., Leon, P.G., Almuhimedi, H., Kelley, P.G., Mugan, J., Acquisti, A., Cranor, L.F., and Sadeh, N., "Nudging Users Towards Privacy on Mobile Devices", ACM Conference on Human Factors in Computing Systems, 2011

[12] Bansal, G., Zahedi, F.M., and Gefen, D., "The impact of personal dispositions on information sensitivity, privacy concern and trust in disclosing health information online", Decision Support Systems, 49(2), 2010, pp. 138-150.

[13] Becker, M., Kolbeck, A., Matt, C., and Hess, T., "Understanding the Continuous Use of Fitness Trackers: A Thematic Analysis", 21. Pacific Asia Conference on Information Systems, 2017

[14] Bélanger, F., and Crossler, R.E., "Privacy in the Digital Age: A Review of Information Privacy Research in Information Systems", MIS Quarterly, 35(4), 2011, pp. 10171041.

[15] Childers, T.L., Houston, M.J., and Heckler, S.E., "Measurement of Individual Differences in Visual Versus Verbal Information Processing", Journal of Consumer Research, 12(2), 1985, pp. 125-154.

[16] Cronbach, L.J., "Coefficient alpha and the internal structure of tests", psychometrika, 16(3), 1951, pp. 297-334.

[17] Dinev, T., and Hart, P., "An Extended Privacy Calculus Model for E-Commerce Transactions", Information Systems Research, 17(1), 2006, pp. 61-80.

[18] Dinev, T., Mcconnell, A.R., and Smith, H.J., "Research Commentary-Informing Privacy Research Through Information Systems, Psychology, and Behavioral Economics: Thinking Outside the "APCO" Box", Information Systems Research, 26(4), 2015, pp. 639-655.

[19] Felder, R.M., and Spurlin, J., "Applications, Reliability and Validity of the Index of Learning Styles", International Journal of Engineering Education, 21(1), 2005, pp. 103-112.
[20] Frederick, S., "American Eocnomic Association", The Journal of Economic Perspectives, 19(4), 2005, pp. 25-42.

[21] Gefen, D., Karahanna, E., and Straub, D.W., "Trust and TAM in online shopping: An integrated model", MIS Quarterly, 27(1), 2003, pp. 51-90.

[22] Hauser, J.R., Urban, G.L., Liberali, G., and Braun, M., "Website Morphing", Marketing Science, 28(2), 2009, pp. 202-223.

[23] Hayes, J., and Allinson, C.W., "Cognitive Style and the Theory and Practice of Individual and Collective Learning in Organizations", Human Relations, 51(7), 1998, pp. 847-871. [24] Hong, W., and Thong, J.Y.L., "Internet Privacy Concerns: An Integrated Conceptualization and Four Empirical Studies", MIS Quarterly, 37(1), 2013, pp. 275-298. [25] Huang, N., Chen, P.-Y., Hong, Y., and Wu, S.-Y., "Digital Nudging for Online Social Sharing: Evidence from A Randomized Field Experiment", 51. Hawaii International Conference on System Sciences, 2018

[26] Kahneman, D., and Tversky, A., "Prospect Theory: An Analysis of Decision under Risk", Econometrica, 47(2), 1979, pp. 263-292.

[27] Keith, M.J., Fredericksen, J.T., Reeves, K.S., and Babb, J., "Optimizing Privacy Policy Videos to Mitigate the Privacy Policy Paradox", 51. Hawaii International Conference on System Sciences, 2018

[28] Kenny, G., and Connolly, R., "Drivers of Health Information Privacy Concern: A Comparison Study", 22. Americas Conference on Information Systems, 2016

[29] Kim, M., and Lennon, S., "The effects of visual and verbal information on attitudes and purchase intentions in internet shopping", Psychology and Marketing, 25(2), 2008, pp. 146-178.

[30] Kirby, J.R., Moore, P.J., and Schofield, N.J., "Verbal and Visual Learning Styles", Contemporary Educational Psychology, 13(2), 1988, pp. 169-184.

[31] Komiak, S.Y., and Benbasat, I., "The effects of personalization and familiarity on trust and adoption of recommendation agents", MIS Quarterly, 2006, pp. 941-960. [32] Laric, M.V., Pitta, D.A., and Katsanis, L.P., "Consumer Concerns for Healthcare Information Privacy: A Comparison of US and Canadian Perspectives", Research in Healthcare Financial Management, 12(1), 2009, pp. 93-111.

[33] Lee, J.-M., and Rha, J.-Y., "Personalization-privacy paradox and consumer conflict with the use of location-based mobile commerce", Computers in Human Behavior, 63(2016, pp. 453-462.

[34] Lee, L., Lee, J., Egelman, S., and Wagner, D., "Information Disclosure Concerns in The Age of Wearable Computing", Proceedings 2016 Workshop on Usable Security, 2016

[35] Li, H., Gupta, A., Zhang, J., and Sarathy, R., "Examining the decision to use standalone personal health record systems as a trust-enabled fair social contract", Decision Support Systems, 57(2014, pp. 376-386.

[36] Li, Y., "The impact of disposition to privacy, website reputation and website familiarity on information privacy concerns", Decision Support Systems, 57(2014, pp. 343-354. [37] Malhotra, N.K., Kim, S.S., and Agarwal, J., "Internet Users' Information Privacy Concerns (IUIPC): The Construct, the Scale, and a Causal Model", Information Systems Research, 15(4), 2004, pp. 336-355. 
[38] Malkin, N., Mathur, A., Harbach, M., and Egelman, S., "Personalized Security Messaging: Nudges for Compliance with Browser Warnings", Proceedings 2nd European Workshop on Usable Security, 2017

[39] Masuda, T., and Nisbett, R.E., "Attending Holistically Versus Analytically: Comparing the Context Sensitivity of Japanese and Americans", Journal of Personality and Social Psychology, 81(5), 2001, pp. 922-934.

[40] Matsunaga, M., "How to Factor-Analyze Your Data Right: Do's, Don'ts, and How-To's", International journal of psychological research, 3(1), 2010,

[41] Mayer, R.E., and Massa, L.J., "Three Facets of Visual and Verbal Learners: Cognitive Ability, Cognitive Style, and Learning Perference", Journal of Educational Psychology, 95(4), 2003, pp. 833-846.

[42] Mcdonald, A.M., and Cranor, L.F., "The Cost of Reading Privacy Policies", Journal of Law and Policy for the Information Society, 4(3), 2008, pp. 543-568.

[43] Mcelroy, J.C., Hendrickson, A.R., Townsend, A.M., and Demarie, S.M., "Dispositional Factors in Internet Use: Personality versus Cognitive Style", MIS Quarterly, 31(4), 2007, pp. 809-820.

[44] Meske, C., and Potthoff, T., "The DINU-model-a process model for the design of nudges", Proceedings of the 25th European Conference on Information Systems (ECIS), Guimarães, Portugal, June 5-10, 2017, 2017, pp. pp. 25872597.

[45] Milne, G.R., Pettinico, G., Hajjat, F.M., and Markos, E., "Information Sensitivity Typology: Mapping the Degree and Type of Risk Consumers Perceive in Personal Data Sharing", Journal of Consumer Affairs, 51(1), 2017, pp. 133-161.

[46] Miltgen, C.L., and Peyrat-Guillard, D., "Cultural and generational influences on privacy concerns: a qualitative study in seven European countries", European Journal of Information Systems, 23(2), 2013, pp. 103-125.

[47] Mirsch, T., Lehrer, C., and Jung, R., "Digital Nudging: Altering User Behavior in Digital Environments", 13. Internationale Tagung Wirtschaftsinformatik, 2017, pp. 634648.

[48] Mishra, A.K., "Organizational responses to crisis", Trust in organizations: Frontiers of theory and research, 261(1996,

[49] Oinas-Kukkonen, H., "A foundation for the study of behavior change support systems", Personal and ubiquitous computing, 17(6), 2013, pp. 1223-1235.

[50] Oulasvirta, A., Suomalainen, T., Hamari, J., Lampinen, A., and Karvonen, K., "Transparency of intentions decreases privacy concerns in ubiquitous surveillance", Cyberpsychology, Behavior, and Social Networking, 17(10), 2014, pp. 633-638.

[51] Riding, R.J., and Taylor, E.M., "Imagery Performance and Prose Comprehension in Seven-year-old Children",
Educational Studies, 2(1), 1976, pp. 21-27. [52] Sadler-Smith, E., "The Role of Cognitive Style in Management Education", Academy of Management Proceedings, 2002(1), 2002,

[53] Schneider, C., Weinmann, M., and Vom Brocke, J., "Digital Nudging - Influencing Choices by Using Interface Design", Communications of the ACM, 2018,

[54] Simon, H.A., "A Behavioral Model of Rational Choice", The Quarterly Journal of Economics, 69(1), 1955, pp. 99118.

[55] Smith, H.J., Dinev, T., and Xu, H., "Information Privacy Research: An Interdisciplinary Review", MIS Quarterly, 35(4), 2011, pp. 989-1015.

[56] Solomon, B.A., and Felder, R.M., "Index of Learning Styles", 1991,

[57] Song, H., and Schwarz, N., "If it's difficult to pronounce, it must be risky: Fluency, familiarity, and risk perception", Psychological Science, 20(2), 2009, pp. 135-138.

[58] Sutanto, J., Palme, E., Tan, C.-H., and Phang, C.W., "Addressing the Personalization-Privacy Paradox: An Empirical Assessment from a Field Experiment on Smartphone Users", MIS Quarterly, 37(4), 2013, pp. 11411164.

[59] Székely, N., Weinmann, M., and Vom Brocke, J., "Nudging People to Pay CO2 Offsets - The Effect of Anchors in Flight Booking Processes", 24. European Conference on Information Systems, 2016

[60] Thaler, R.H., and Sunstein, C.R., Nudge: Improving Decisions About Health, Wealth, and Happiness, 2008.

[61] Tsai, J.Y., Egelman, S., Cranor, L., and Acquisti, A., "The Effect of Online Privacy Information on Purchasing Behavior: An Experimental Study", Information Systems Research, 22(2), 2011, pp. 254-268.

[62] Wang, H.-C., and Doong, H.-S., "Online customers' cognitive differences and their impact on the success of recommendation agents", Information \& Management, 47(2), 2010, pp. 109-114.

[63] Weinmann, M., Schneider, C., and Brocke, J.V., "Digital Nudging", Business \& Information Systems Engineering, 58(6), 2016, pp. 433-436.

[64] Whittlesea, B.W., and Williams, L.D., "Why do strangers feel familiar, but friends don't? A discrepancyattribution account of feelings of familiarity", Acta psychologica, 98(2-3), 1998, pp. 141-165. 